\title{
BRAVO - Sistema Web de Apoio à Pesquisa em Educação
}

\author{
Raphael Alex de Sousa ${ }^{1}$, Luciano Édipo P. da Silva ${ }^{2}$ \\ ${ }^{1}$ Formando do Curso de Sistemas de Informação - Universidade Federal de Mato \\ Grosso do Sul (UFMS) \\ Av. Rio Branco, 1.270 - 79.304-902 - Corumbá - MS - Brazil \\ ${ }^{2}$ Professor Assistente do Curso de Sistemas de Informação - Universidade Federal de \\ Mato Grosso do Sul (UFMS) \\ raphael.crba@gmail.com, luciano.silva@ufms.br
}

\begin{abstract}
The aim of this paper is to describe the importance of quantitative research methods using databases, also describes the steps taken for the development of a free tool-BRAVO. The tool offers, in a simplified way, the generation of dynamic filters and reports for educational researchers, using as the source of knowledge extraction the databases and microdata provided by Instituto Nacional de Estudos e Pesquisas em Educacionais Anísio Teixeira (INEP), timeline 2007-2014.
\end{abstract}

Resumo. $O$ objetivo deste trabalho é expor a importância dos métodos de pesquisa quantitativos utilizando-se bancos de dados assim como apresentar o desenvolvimento de uma ferramenta livre de apoio à pesquisa em educação BRAVO. A ferramenta oferece, de forma simplificada, a geração de filtros dinâmicos e relatórios gerenciais para pesquisadores da área da educação e afins, utilizando-se da extração de conhecimento alimentado pela fonte de microdados disponibilizados pelo Instituto Nacional de Estudos e Pesquisas em Educacionais Anísio Teixeira (INEP), do espaço temporal 2007-2014.

\section{Introdução}

A educação e seu emprego, ao longo do tempo, apresentam-se como umas das mais valiosas fontes de evolução para o ser humano. Atualmente, cada vez mais nos deparamos com dados que explicitam situações alarmantes em relação à educação, dados como: níveis de letramento, quantidade de analfabetos funcionais, entre outros. Há um nicho de profissionais e pesquisadores que dedicam seu tempo e esforços na obtenção de conhecimento em bases de dados relacionadas a educação. São pesquisas em diversas subáreas, tais como: aplicação de políticas públicas, medição de ações governamentais, entre outras. Frente a isso, há cada vez mais dados, relacionados a educação que são disponibilizados formando grandes bases de dados. Tais fatores exigem que haja a necessidade de recursos computacionais para que se consiga executar a extração de conhecimento ou um simples grupamento de dados.

No Brasil, uma das maiores base de dados relacionada a educação é a base de dados oferecida pelo Instituto Nacional de Estudos e Pesquisas Educacionais Anísio Teixeira INEP. Todos os anos o INEP disponibiliza, os dados que são coletados via formulários, 
na forma de microdados ${ }^{1}$, relacionados a educação nacional abrangendo todas as suas fases: Educação Básica (1995-2012), Educação Superior (1995-2011), entre outras. Esses dados, nativamente, são disponibilizados como inputs de dois programas de inteligência analítica, o Statistical Analysis System (SAS) e Statistical Product and Service Solutions (SPSS), cujas licenças são pagas, o que dificulta o acesso a estas ferramentas pelo grande público.

Ainda nessa linha de raciocínio, sobre as grandes bases de dados geradas e disponibilizadas, além das dificuldades dos pesquisadores na extração de conhecimento, é importante se verificar também a validade, fidedignidade e consistência dos dados. Para alcançar seu objetivo, este trabalho analisou os dados disponibilizados pelo INEP, pois tais bancos de dados, em teoria, são de grande valia para as pesquisas em educação uma vez que abrangem dados sobre todo o processo de escolarização em uma escala nacional.

Além das informações supracitadas, os profissionais que têm acesso a essas bases de dados e ferramentas automatizadas para realização de suas pesquisas, muitas vezes não dispõem de conhecimento técnico suficiente, experiência vivenciada no dia a dia do projeto $^{2}$, tampouco tempo para o aprendizado da ferramenta, ora pela complexidade da ferramenta ou por sua falta de familiaridade com recursos computacionais. Esses fatos acabam, por muitas vezes, inviabilizando ou tornando o processo investigatório das pesquisas educacionais um processo moroso.

Os problemas que foram percebidos e que os pesquisadores em educação vivenciaram foram: falta de conhecimento técnico em recursos computacionais, dificuldade em extração de conhecimento em grandes bases de dados, bases de dados ilegíveis e dificuldade de normalização de dados. A principal finalidade do BRAVO é mitigar as dificuldades em extração de conhecimento das bases de dados do INEP pelos profissionais pesquisadores, tornando mais simples e menos propensa a erros a utilização das bases de dados disponíveis.

O modelo de desenvolvimento utilizado na construção da ferramenta BRAVO foi o modelo cascata, que abrange todas as fases da concepção de uma aplicação: comunicação, planejamento, modelagem, construção e manutenção. Juntamente ao modelo cascata utilizou-se conceitos específicos de projetos de webapps ou simplesmente sistemas para web. O BRAVO foi planejado e desenvolvido para ser uma ferramenta web. Tal característica traz mais versatilidade e acessibilidade para os usuários. Essas características, dentre as que analisamos, foram as que mais influenciaram na escolha e junção dos dois modelos de desenvolvimento cascata e webapp [Pressman 2011].

\footnotetext{
${ }^{1}$ Respostas individuais em pesquisas ou censos.

${ }^{2}$ Antes da elaboração deste trabalho, as dificuldades que foram percebidas e citadas em trechos anteriores, na participação no grupo de pesquisa em educação na Universidade Federal de Mato Grosso do Sul campus do Pantanal, especificamente no projeto A Escolarização de Alunos com Deficiência e Rendimento Escolar: uma análise dos indicadores educacionais em municípios brasileiros. Esse projeto é interinstitucional e está focado na realização de pesquisas em diversas áreas da educação. Neste campus em especial, o projeto concentra seus esforços na análise de dados quantitativos para posterior análise qualitativa. $\mathrm{O}$ projeto investiga áreas como educação especial, políticas públicas e pedagogia social, entre muitas outras.
} 
CBIE-LACLO 2015

Anais dos Workshops do IV Congresso Brasileiro de Informática na Educação (CBIE 2015)

\section{A importância da utilização dos dados quantitativos para a pesquisa em educação}

Iniciamos a fundamentação sobre a importância de pesquisas em educação utilizando de métodos quantitativos, apresentando a fala de [Gatti 2004]: "Atualmente, na área da pesquisa educacional, excluindo análises de dados de avaliações de rendimento escolar realizadas em alguns sistemas educacionais no Brasil, poucos estudos empregam metodologias quantitativas", fato que nos remete a uma inegável falta de familiaridade frente aos métodos de pesquisa e análise quantitativas, suas principais fontes de informação, no caso, os dados, tanto quanto suas possibilidades. Segundo [Choo 2003] "O pesquisador que conhece as bases de dados, sua estrutura e os recursos de busca pode obter vantagem dos sistemas de informação on-line.", palavras que são vistas como de grande valia para corroborar o fato que [Gatti 2004] nos trouxe anteriormente. Com os avanços tecnológicos, especialmente falando sobre a disponibilização de conteúdo via internet, e com a oferta de cada vez mais bases de dados especializadas para se analisar, os pesquisadores e agentes envolvidos em pesquisas não podem simplesmente ignorar os métodos quantitativos, pois, como [Choo 2003] salienta "[...] há problemas educacionais que para sua contextualização e compreensão necessitam ser qualificados através de dados quantitativos".

$\mathrm{Na}$ argumentação sobre a geração das bases de dados e os problemas enfrentados trazemos também [Garcia 2013] que complementa: "É de conhecimento geral que numa vasta gama de domínios, tanto científicos como empresariais, a quantidade de dados recolhidos e processados é muitas vezes gigantesca. É, portanto muito difícil, senão mesmo impossível para analistas humanos, efetuar uma análise adequada em tão elevada quantidade de dados e mesmo para um especialista é difícil identificar informação valiosa implícita em tantos dados ou que traga novo conhecimento para o domínio". A BRAVO surge exatamente para apoiar os utilizadores na etapa de processamento dos dados na quantificação, grupamento, separação, entre outros.

Portanto, tais análises podem substanciar pesquisas e possivelmente guiá-las para informações até então não identificadas. Esse método de pesquisa quando empregado em alguma investigação é normalmente complementado por outros métodos de análise, tal como o qualitativo, pesquisa - ação e tantos outros. É inegável que o referido método não é plenamente empregado, ora por falta de ferramentas acessíveis, ora por falta de conhecimento, ou até mesmo, pela simples descrença na veracidade de tais dados, como [Choo 2003] afirma: "Essa dificuldade no uso de dados numéricos na pesquisa educacional rebate de outro lado na dificuldade de leitura crítica, consciente, dos trabalhos que os utilizam, o que gera na área educacional dois comportamentos típicos: ou se acredita piamente em qualquer dado citado (muitas vezes dependendo de quem cita - argumento de autoridade), ou se rejeita qualquer dado traduzido em número por razões ideológicas reificadas, a priori".

Esse ceticismo e aversão sobre a utilização dos dados, sejam eles, por exemplo, fornecidos pelo Instituto Brasileiro de Geografia e Estatística (IBGE), INEP ou outras bases quaisquer disponibilizadas em domínio público para ampla utilização, pode partir do obscuro e nebuloso sistema de coleta, organização, avaliação dos dados até sua posterior disponibilização. Nesta linha de raciocínio [Assis 2007] tece a seguinte fala 


\section{CBIE-LACLO 2015}

Anais dos Workshops do IV Congresso Brasileiro de Informática na Educação (CBIE 2015)

sobre a confiança das informações "Algumas vezes, tem-se dificuldade em avaliar a confiabilidade da informação, em razão do fato de a fonte ou a reputação da instituição que a fornece ser desconhecida.”. Acreditamos que não é o caso do INEP, todavia, traçando o processo geral que os órgãos adotam da coleta à disponibilização dos dados, destacamos, sem dúvida, a importância de estes receberem atenção permanente quanto à sua coleta, organização, avaliação, validação e disponibilização, isso segundo [Ferreira 2003] aumentará o campo de ação das instituições provedoras de dados, exemplificamos com os dados e casos do INEP e IBGE além de tantas outras.

Como argumento adicional para o ceticismo sobre a utilização e qualidade dos dados trazemos também a fala de [Ferraro 2002] "[...] o problema da qualidade (validade e fidedignidade) dessas estatísticas, problema este certamente tão velho quanto as próprias estatísticas, provavelmente mais grave quando se trata de estatísticas sociais, como as da educação.”, por isso, neste trabalho descrevemos as bases de dados do INEP para que o pesquisador a conheça e assim decida utilizá-las ou não.

Frente a esse cenário de falta de adeptos dos dados em pesquisas de cunho quantitativo, normalmente o pesquisador em educação que opta pela utilização de tal, a tem utilizado em conjunto a outras técnicas para análise de dados. [Choo 2003] na mesma linha argumenta: "Os métodos de análise de dados que se traduzem por números podem ser muito úteis na compreensão de diversos problemas educacionais. Mais ainda, a combinação deste tipo de dados com dados oriundos de metodologias qualitativas, podem vir a enriquecer a compreensão de eventos, fatos, processos".

\section{Bases de Dados do INEP}

Como a matéria-prima mais preciosa da ferramenta aqui proposta temos os dados disponibilizados pelo INEP. Esses dados após o processo de análise e normalização foram incorporados a um sistema que vislumbra dar ao usuário, como funcionalidade principal, a geração de filtros e relatórios gerenciais como melhor se ajuste às suas pesquisas e empreendimentos. Como a ferramenta aqui descrita tem como principal componente essas bases de dados, nesta seção apresentamos as características nativas encontradas neste componente, bem como explicitamos as etapas realizadas para seu beneficiamento e lapidação. Por fim, descrevemos a integração do conjunto de dados em um Sistema Gerenciador de Banco de Dados. O SGBD, segundo [Silberschatz 1999] propicia "[...] um ambiente tanto conveniente quanto eficiente para a recuperação e armazenamento das informações do banco de dados". Ao final deste ciclo, as bases de dados que foram integradas ao SGBD, e serão um suporte sólido e confiável a ferramenta.

\subsection{Comunicação}

Nesta seção partilhamos o processo de aquisição das bases de dados e também o levantamento das características do banco de dados disponibilizados pelo INEP. A captação do arquivo compactado que contém a base de dados é bem simples, basta se entrar no sítio do INEP - http://portal.inep.gov.br/home - navegar até a seção Informações Estatísticas e posteriormente Microdados. Nesta seção nos deparamos com os pacotes de dados que estão disponíveis para download, além de algumas informações 


\section{CBIE-LACLO 2015}

Anais dos Workshops do IV Congresso Brasileiro de Informática na Educação (CBIE 2015)

complementares sobre as bases de dados como formato do banco, os softwares que as mesmas podem ser utilizadas e etc.

Após a aquisição do arquivo compactado com as bases que escolhemos para o trabalho e prototipação da ferramenta - censo escolar 2012 - denominadas pela instituição INEP como "Microdados Censo Escolar", identificamos que além da pasta DADOS contendo as bases de dados, o INEP disponibilizou também duas outras pastas: a INPUTS e LEIA-ME. A pasta INPUTS contém diversos arquivos com extensões .sas e .sps. Estes, são arquivos de inicialização e leitura das bases de dados de dois softwares estatísticos o SAS e o SPSS.

Continuando a explanação pela pasta de arquivos, encontramos também uma pasta chamada LEIA-ME, onde foram disponibilizados os formulários aplicados na captação dos dados denominados como 'GABARITO', pois estão preenchidos e, servem como exemplo de preenchimento. O INEP disponibilizou também, um dicionário de dados que segundo [Lauesen 2002] é uma descrição verbal de dados estruturado de forma sistemática e, ainda complementa que é excelente para especialistas bem como usuários. Sua importância é reconhecida por [Silberschatz 1999]. Ele argumenta que um sistema de banco de dados precisa manter informações sobre as relações. Essa informação é chamada por ele, além de dicionário de dados, de "catálogo do sistema".

No dicionário há informações como: uma apresentação, os objetivos e também sobre o formato em que as bases são disponibilizadas. Por fim, nesta parte introdutória, o documento apresenta os dizeres: "[...] por meio dessas informações, é possível obter um amplo panorama da educação brasileira que inclui cerca de 350 variáveis para quase 50,5 milhões de matrículas, mais de 2 milhões de docentes e, aproximadamente, 193 mil estabelecimentos; indubitavelmente, um rico acervo sobre a educação básica brasileira e uma fonte segura e eficaz de obtenção de dados, acessíveis aos pesquisadores, estudantes, gestores e demais da sociedade."

O trecho acima demonstra a grande significância dessas bases de dados para este trabalho e, mais importante ainda, para a sociedade brasileira, pois extraindo conhecimento das mesmas é possível, por exemplo, aplicar políticas específicas para alguma área com alto índice de evasão escolar, prever a possível falta de professores, monitorar o progresso dos alunos e uma infinidade de outras possibilidades, por isso resolvemos exibi-lo. Com as informações explicitadas sobre os dados e variáveis, não é possível prever a vasta gama de aplicações que os dados podem ter o que é, neste caso, uma excelente constatação. A partir daqui realizamos o processo de ajuste destas bases para sua posterior integração ao SGBD.

\subsection{Visão Geral das bases}

Após o processo de captação, descrição e normalização das bases podemos ter uma ideia geral de quais características ela apresenta assim que são adquiridas. Os dados são disponibilizados em pastas separadas por categorias e com nomeação intuitiva, o que é um ótimo fator para diminuir a redundância dos dados, além de ser muito bom para a identificação dos dados a serem trabalhados. Ainda dentro de cada uma das pastas separadas por categoria há ainda a separação por estado, o que acaba minimizando o problema do isolamento dos dados de uma mesma região. 


\section{CBIE-LACLO 2015}

Anais dos Workshops do IV Congresso Brasileiro de Informática na Educação (CBIE 2015)

Um dos pontos negativos que pode ser identificado é a utilização de ferramentas com licença comercial para a extração de conhecimento das bases de dados - SAS e SPSS. O SAS e o SPSS são softwares estatísticos que em sua essência são projetados para servir como soluções corporativas de inteligência analítica. Inteligência analítica é segundo está exposto no Portal [Sas 2015] "[...] o conjunto de processos, modelos e tecnologias que ajuda empresas de diferentes setores da economia a entender em profundidade os próprios negócios e também os mercados em que atuam". Depois de analisar o contexto dos dados do INEP, entendemos então, que estas ferramentas têm por objetivo ajudar na descoberta de conhecimento nas bases de dados, pois segundo o [Sas 2013] a inteligência analítica dá a possibilidade de transformação das bases de dados armazenadas em informações estratégicas com os cenários pré-determinados pelos usuários. Não entramos no mérito de comparação de funcionalidades da ferramenta aqui proposta e os softwares utilizados nativamente pelo INEP, pois acreditamos que tenha ficado explícito que ambas as soluções são utilizadas para tratamento dos dados, entretanto, são completamente diferentes em ambiente de execução, plataforma e funcionalidades.

\section{Desenvolvimento}

Para o desenvolvimento deste trabalho utilizamos o modelo cascata, ou ciclo de vida clássico de desenvolvimento de software, que é uma abordagem sistemática e sequencial segundo [Pressman 2011]. Esse modelo além de ser uma das abordagens mais antigas ainda é amplamente usado na engenharia de software conforme o autor.

A escolha do modelo que seguimos e da bibliografia por [Pressman 2011], deu-se pela lógica de fácil entendimento e execução do método que o autor expõe em seu texto sobre no modelo cascata. Todavia, não podemos deixar de salientar a importância de se consultar diversas bibliografias para uma melhor compreensão do domínio do problema, portanto, foi consultada também, após a escolha do modelo, a obra de [Sommerville 2011] para complementar o entendimento sobre alguns conceitos e procedimentos, entre muitos outros.

Juntamente a escolha do modelo principal a ser seguido, escolhemos também para sua complementação, em outro paradigma, técnicas que foram utilizadas de forma harmoniosa junto ao modelo cascata. Estas técnicas são relacionadas a um projeto de webapp. Isso se fez necessário pela escolha das tecnologias de desenvolvimento e do ambiente de execução que estão relacionadas à web. Essa junção de paradigmas segundo [Pressman 2011] pode e deve ser realizada, pois tendem a potencializar suas características em um único projeto.

Para a garantia de qualidade do BRAVO pesquisamos algumas características essenciais descritas nas bibliografias consultadas e que, por sua importância, foram abordadas neste trabalho. Conforme [Pressman 2011] a simplicidade, consistência, identidade, navegabilidade, apelo visual e a compatibilidade são algumas dessas características. Foram levados também em consideração alguns fatores que são relacionados à qualidade de uma aplicação web que segundo [Sommerville 2011] são - usabilidade, funcionalidade, confiabilidade, eficiência e facilidade de manutenção. Finalmente, como extensão dos atributos ligados as qualidades supracitadas, utilizamos também os que 
CBIE-LACLO 2015

Anais dos Workshops do IV Congresso Brasileiro de Informática na Educação (CBIE 2015)

[Olsina 1999], traz que são - segurança, disponibilidade, escalabilidade e time-tomarket $^{3}$.

\subsection{Comunicação}

Uma das partes mais importantes do projeto, pois, é nesta fase que coletamos quais as funcionalidades que serão implementadas, quais os requisitos funcionais e nãofuncionais entre diversas outras coisas. É importante destacar que nesta fase de comunicação é que tivemos uma maior interação com os clientes do projeto (stakeholders ${ }^{4}$ ), no caso os pesquisadores em educação. Essa fase foi crucial para o sucesso do projeto de software como um todo pois foi aqui que realmente entendemos o que eles necessitavam.

\subsection{Planejamento}

Fase em que montamos todas as estimativas e o cronograma de acompanhamento do projeto de software. O principal objeto desta fase foi traçar uma rota para o projeto, identificar seus riscos e os recursos necessários [Pressman 2011].

\subsection{Modelagem}

Nesta etapa iniciamos o trabalho em equipe como um todo. Foram abordados quatro passos nesta etapa: definição das estruturas de dados que serão utilizadas, concepção da arquitetura do software, detalhamento dos procedimentos a serem empregados e por fim a caracterização da interface seguindo o que expõe [Pressman 2011]. Ao final desta fase, toda a documentação sobre as funcionalidades, arquiteturas, procedimentos e demais, ficaram prontas e serviram de base para a fase posterior: desenvolvimento. A documentação foi um dos primeiros deliverables ${ }^{5}$ do projeto e teve a função primordial de guiar os desenvolvedores na produção da ferramenta. $\mathrm{Na}$ fase de modelagem, junto com o modelo cascata, empregamos conceitos de um projeto de webapp, com as fases de projeto: de interface, estético, do conteúdo, de navegação, arquitetural e de componentes.

\subsubsection{Projeto de Interface}

O foco desta parte no projeto de um webapp foi a construção de uma interface gráfica layout - que foi a principal forma de interação humano-computador. Esta fase subdividiu-se em quatro outras: análise de interfaces, projeto de interfaces, construção da interface e por fim a validação da interface. Esta fase também caracterizou-se por ter um ciclo espiral com mudanças em cada iteração segundo [Pressman 2011]. Das características já citadas que visam a qualidade de projetos deste tipo, a que se apresenta nesta fase do processo é a usabilidade.

\footnotetext{
${ }^{3}$ Tempo de produção até a comercialização do produto.

${ }^{4}$ Pessoas envolvidas no projeto: analistas, desenvolvedores, clientes, usuários finais, etc.Pessoas envolvidas no projeto: analistas, desenvolvedores, clientes, usuários finais, etc.

${ }^{5}$ Itens que podem ser entregues ao cliente. Exemplo: relatório com análise de requisitos, diagramas, etc.
} 
CBIE-LACLO 2015

Anais dos Workshops do IV Congresso Brasileiro de Informática na Educação (CBIE 2015)

\subsubsection{Projeto Estético}

Nesta fase pensamos em formas de tornar a aplicação atraente aos olhos dos usuários. Basicamente utilizamos técnicas e elementos de design em sua elaboração - cores, tipografias, formas, programas gráficos, fotografia, entre outras. Podemos citar duas das características relacionadas a qualidade: a identidade e o apelo visual. Estas características foram empregadas utilizando-se as técnicas do profissional designer gráfico que geralmente criam os layouts, seções e interfaces gráficas da aplicação.

\subsubsection{Projeto de Conteúdo}

Nesta etapa desenvolvemos os esquemas relacionais do conteúdo a que foi contemplado pelas diversas seções além das formas de relacionamento entre eles. Foram também pensadas as informações que cada uma das seções ou representações de conteúdos terão. Uma das características mais marcantes que o projeto tende a adquirir nesta etapa é a consistência.

\subsubsection{Projeto de Navegação}

Nesta parte do projeto de um webapp, teve como principal objetivo, a criação da semântica e sintaxe de navegação. $\mathrm{Na}$ semântica buscamos identificar e traçar a hierarquia de navegação entre os diversos perfis de usuários. Já na sintaxe propomos as formas para que essa navegação flua utilizando abordagens como a utilização de links, ícones, barras de navegação etc. Esta fase visou ajudar o usuário a compreender com facilidade a aplicação, ou seja, deve se atentar ao conceito da simplicidade e navegabilidade, pois a navegação precisa ser o mais amigável possível ao usuário.

\subsubsection{Projeto Arquitetural}

Essa fase ditou como o conteúdo geral da aplicação está disposto para o usuário final. Aqui lidamos com uma característica nítida aos usuários e sua interação com a ferramenta final, a navegabilidade. Nesta fase buscamos agregar à ferramenta, a eficiência, pois é nesta organizamos todo o conteúdo com foco no objetivo fim da ferramenta. Por outro lado, esta fase arquitetural trouxe ao BRAVO características como segurança e facilidade de manutenção com nossa opção pela arquitetura $\mathrm{MVC}^{6}$. Tais características são obtidas pelo uso de encapsulamento da aplicação e pela própria implementação do modelo que visa a separação dos scripts em camadas distintas.

\subsubsection{Projeto de Componentes}

Todo sistema web ou webapp herda todas as características naturais de um software convencional. Com o avanço do ambiente web, a maximização e sofisticação de processamento são necessárias, pois segundo [Pressman 2011] "O projeto de componente define as estruturas de dados, os algoritmos, as características das interfaces e os mecanismos de comunicação alocados a cada componente de software". Em outras palavras, como cada um dos módulos do sistema deve funcionar e sua integração. Abaixo pode ser visto o modelo final dos componentes do BRAVO:

\footnotetext{
${ }^{6}$ Modelo, visão e controle.
} 


\section{CBIE-LACLO 2015}

Anais dos Workshops do IV Congresso Brasileiro de Informática na Educação (CBIE 2015)

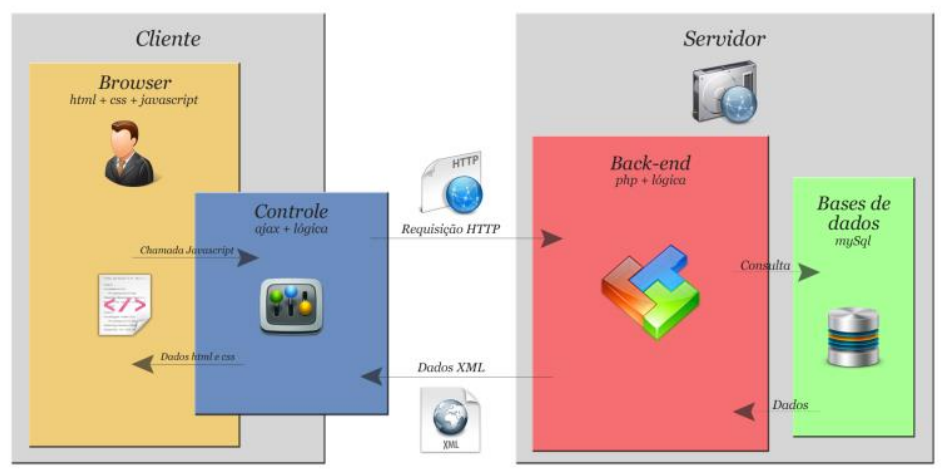

Figura 1. Diagrama de Componentes

Aqui abordamos características específicas de conceitos como funcionalidade, confiabilidade, disponibilidade entre outras.

\subsection{Construção}

Nesta fase realizamos a produção propriamente dita. Essa fase foi dividida em duas grandes fases: codificação e testes. A fase de codificação foi onde toda a parte lógica da aplicação foi pensada e produzida. Na codificação descrevemos ainda qual tecnologia utilizamos para a construção da ferramenta bem como o paradigma de programação escolhido. Já na fase de testes descrevemos quais foram os testes executados ao longo da construção da aplicação bem como a intenção da utilização do mesmo.

\subsection{Manutenção}

É a última fase do ciclo fundado no método cascata. A partir desta fase o BRAVO foi entregue para a utilização pelo cliente. Por fim, na busca da garantia de satisfação do cliente e também para o aprimoramento da ferramenta aqui proposta, ofereceremos os serviços de suporte, além de buscar o feedback em relação ao produto por parte do cliente. É a última fase do ciclo fundado no método cascata. A partir desta fase o BRAVO foi entregue para a utilização pelo cliente. Por fim, na busca da garantia de satisfação do cliente e também para o aprimoramento da ferramenta aqui proposta, ofereceremos os serviços de suporte, além de buscar o feedback em relação ao produto por parte do cliente.

\section{Considerações Finais}

Atualmente o BRAVO encontra-se na fase de testes, fase que já obtivemos resultados significantes com a entrega do primeiro protótipo funcional ${ }^{7}$, embora ele ainda retorne apenas resultados primitivos das consultas realizadas no banco de dados.

Após o início do desenvolvimento, identificamos a necessidade da elaboração de uma função de erro para cada uma das consultas realizadas, haja vista, que o banco de dados tem muitas falhas nativas. Ao identificarmos essas falhas na análise das bases de dados, as mesmas serão apresentadas para os usuários, desta forma a decisão sobre a utilização dos dados obtidos nos resultados dos filtros em sua mão. Constatamos que as bases

\footnotetext{
${ }^{7}$ Link de acesso: bravo.sek.com.br/app
} 


\section{CBIE-LACLO 2015}

Anais dos Workshops do IV Congresso Brasileiro de Informática na Educação (CBIE 2015)

sofrem muitas alterações de ano a ano, o que, sem dúvida, dificultou a implementação de funções de comparação temporal.

Embora as estimativas fossem um pouco otimistas prevendo a finalização da ferramenta, no decorrer do projeto encontrou-se diversos percalços que fez com que os prazos mudassem. Como resultados e deliverables, já entregues, destacamos o documento de especificação de requisitos e o protótipo de interface funcional como pode ser observado no apêndice deste trabalho.

Acreditamos, analisando os feedbacks apresentados pelos envolvidos, que a ferramenta supriu as expectativas e também contemplou todos os requisitos previamente fornecidos pelos pesquisadores.

Como projetos futuros, seria interessante a implementação de funções temporais, uma função de cálculo de erro por filtro e a apresentação de uma outra estrutura do banco de dados a fim de facilitar o manuseio dos dados.

\section{Referências}

Assis, W. M. Fontes de informação para o setor siderúrgico. Revista Ciência da Computação, v. 36, n. 2, 2007. http://ref.scielo.org/jzz5wb. Acessado em 21 de maio de 2015.

Choo, C. A organização do Conhecimento: como as organizações usam a informação para criar significado, construir conhecimento e tomar decisões. Editora Senac, São Paulo, 2003.

Elmasri, R. Sistemas de banco de dados, Pearson Addison Wesley, São Paulo, 2011.

Ferraro, A. R. Analfabetismo e Níveis de Letramento no Brasil: o que dizem os censos? Educação Social, volume 23, páginas 21-47, 2002.

Ferreira, S. P. Produção e Disponibilização de Estatísticas: uma abordagem institucional. São Paulo em Perspectiva, páginas 17-25, 2003.

Garcia, J. M. E. Assistente Web para Análise Automática e Colaborativa de Dados Webcad. Tese de Mestrado. Universidade do Porto, Porto, 2013.

Gatti, B. A. Implicações e perspectivas da pesquisa educacional no Brasil contemporâneo; Cadernos de Pesquisa, volume 113, páginas 65-81, 2004.

Heuser, C. A. Projeto de Banco de Dados, Sagra Luzzatto, Porto Alegre, 2001.

Lauesen, S. Software requirements: styles and techniques. Addison-Wesley, 2002.

Olsina, L. Specifying quality characteristics and attributes for websites. 1St ICSE Workshop on Web Engeneering, 1999.

Pressman, R. Engenharia de Software: uma abordagem profissional, AMGH, Porto Alegre, 2011.

Portal SAS. Statistical analysis system, 2015. Acessado em 21 de maio de 2015.

Silberschatz, A; Korth, H. F; Sudarshan, H. F Sistema de Banco de Dados. Pearson Makron Books, 1999. 